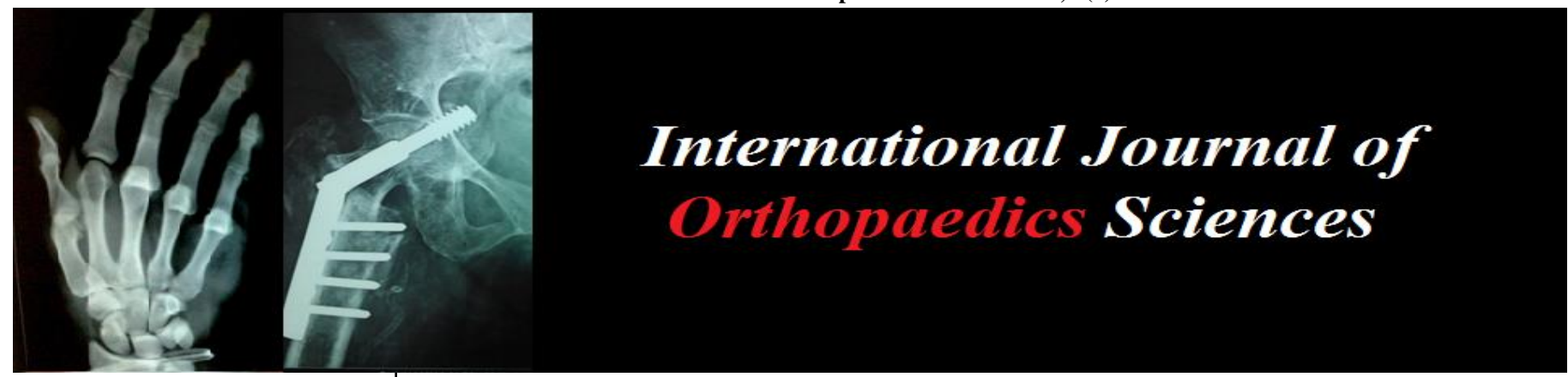

ISSN: $2395-1958$

IJOS 2017; 3(4): 319-323

(C) 2017 IJOS

www.orthopaper.com

Received: 08-08-2017

Accepted: 12-09-2017

Dr. Hari Prasad S

Assistant professor, Department of Orthopaedics, RLJH, Kolar,

Karnataka, India

Dr. Siddaram N Patil

Professor, Department of

Orthopaedics, RLJH, Kolar,

Karnataka, India

Dr. Sarath Chandra $P$

Resident, Department of

Orthopaedics, RLJH, Kolar,

Karnataka, India

Dr. Cecil Fernando A

Resident, Department of

Orthopaedics, RLJH, Kolar,

Karnataka, India
Correspondence

Dr. Hari Prasad S

Assistant professor, Department

of Orthopaedics, RLJH, Kolar,

Karnataka, India

\section{Functional outcome of unstable intertrochanteric femur fracture in elderly osteoporotic patients treated by primary cemented bipolar hemiarthroplasty versus internal fixation with proximal femoral nailing}

\author{
Dr. Hari Prasad S, Dr. Siddaram N Patil, Dr. Sarath Chandra P, Dr. Cecil \\ Fernando A
}

DOI: https://doi.org/10.22271/ortho.2017.v3.i4e.43

\begin{abstract}
Backround: Intertrochanteric fractures in the elderly pose certain special problems and need to be addressed in a balanced way as on one hand senile osteoporosis requires prolonged immobilization, on the other hand rapid mobilization is required as they are generally medically compromised due to age and associated diseases. Despite several randomized trials and comparative studies treatment of unstable intertrochanteric fractures in elderly patients is still controversial, which we want to investigate in this set-up which caters to a vast rural population.

Methods: A retrospective analysis of 54 patients, of which 27 each were operated with cemented bipolar hemiarthroplasty (BPA) and proximal femur nail (PFN) between January 2012 till July 2016 was done. Primary outcome was assessed in both groups using Harris hip score( HHS) at 4weeks, 6 weeks, 12 weeks, 6months and 1 year on outpatient basis. The secondary outcome measures were intraoperative blood loss, transfusion rate, surgical time, bed to chair time, chair to walking time, limb length discrepancy at final follow up. All complications were noted at follow ups.

Results: The HHS was significantly higher for BPA group at all follow ups, at 4 weeks of follow up was 77.81 for BPA and 71.18 for PFN (p-value $=0.001$ ) and at 1 year of follow up was 85.55 for BPA and 77.03 for PFN (p-value $=0.001)$. Statistically significant differences were found between BPA and PFN groups with reference to intraoperative blood loss, transfusion rate, surgical time, bed to chair time, chair to walking time, limb length discrepancy at final follow up.

Conclusion: The bipolar hemiarthroplasty group had fewer complications and earlier mobilization with better Harris hip score at all follow ups, which indicate bipolar hemiarthroplasty is a better option in the treatment of unstable intertrochanteric fractures in senile osteoporotic patients.
\end{abstract}

Keywords: unstable intertrochanteric fractures, Bipolar hemiarthroplasty (BPA), Proximal femur nailing (PFN), Senile osteoporosis, Harris hip score (HHS)

\section{Introduction}

The incidence hip fractures is approximately 80 per 100,000 persons and is expected to double over the next 50 years as the population ages and intertrochanteric fractures makes up $45 \%$ of these fractures ${ }^{[1]}$. It is estimated that the number of these fractures is 1.26 million cases annually worldwide, which is expected to amount to 2.6 million cases in 2025 and to 4.5 million cases in 2050. There is, in fact, an increase in the incidence of these fractures in correlation with the aging of the population, especially at age brackets of over 50 and the odds are doubled with a 10-year increase in age ${ }^{[2]}$.

Due to problems caused by these fractures and an increase in the number of the elderly population leading to a significant increase in the incidence of these fractures, it is absolutely necessary to use an effective and appropriate treatment modality for such patients. Many treatment methods have been used for the reduction of intertrochanteric fractures, including dynamic hip screw (DHS), proximal femoral nail (PFN), unipolar and bipolar hemiarthroplasty and external fixation. 
However, it is difficult to achieve and maintain a stable fixation in elderly patients due to their poor bone quality ${ }^{[3]}$. Intertrochanteric fractures in the elderly pose certain special problems. In this age group the fracture configuration is generally comminuted with presence of extensive osteoporosis. There is problem with correct and accurate placement of the implant and hold of the implant hence prolonged immobilization is required to achieve complete union. On the other hand there is a need for rapid full weight bearing mobilization of this group of patients as they are generally medically compromised due to age and associated diseases. In addition, these patients may not have adequate psychomotor skills required for graded and protected weight bearing required with internal fixation. Hence there are two conflicting requirements that need to be addressed to in a balanced way. So despite the publication of reports of randomized trials and comparative studies treatment of unstable intertrochanteric fractures in elderly patients is still controversial ${ }^{[4]}$. In these patients due to combination of osteoporosis and instability, early resumption to full weight bearing is difficult. Because of prolonged immobilization, complications like deep vein thrombosis, hypostatic pneumonia, pressure sores, dehydration, atelectasis, metabolic disturbances, etc are likely. So they have increased the morbidity and mortality threshold ${ }^{[4]}$.

Despite advances in internal fixation techniques, the failure rate of the dynamic hip screw is $6.8 \%$ to $9.8 \%$ while failure rate of the proximal femoral nail is between $7.1 \%$ and $12.5 \%$ in unstable fractures. There have been various reports of successful outcomes after the use of hemiarthroplasty and total hip arthroplasty ${ }^{[5]}$.

Stappaerts et al., in a prospective randomized study, concluded that primary cemented endoprosthesis brought better results than compression hip screw in unstable intertrochanteric fractures in elderly osteoporotic patients who were eligible for early mobilization ${ }^{[6]}$. Parvjeet found in his study that the patients treated with bipolar prosthesis had earlier rehabilitation than those treated with internal fixation, which decreased the overall morbidity, and he therefore concluded that bipolar prosthesis might be favored in oldaged patients even though there was no major difference in the choice of either implant ${ }^{[7]}$.

Hemiarthroplasty and THA, as two possible treatment options for unstable intertrochanteric fractures, may offer the potential for quick recovery with little risk of mechanical failure, avoid the risks often associated with internal fixation, andenable patients to maintain a good level of function immediately after surgery ${ }^{[8]}$. Treatment of unstable intertrochanteric fracture is still controversial, despite of the publication of reports of randomized trials and comparative studies and their role in unstable osteoporotic and severely comminuted intertrochanteric fractures is still to be defined ${ }^{[3]}$.

The purpose of this retroprospective study is to analyze and compare the role of primary cementedbipolar hemiarthroplasty (BPA) and proximal femur nailing (PFN) in cases of unstable osteoporotic intertrochanteric femur fractures in elderly.

\section{Materials \& Methods}

From January 2012 till July 2016 a total of 54 patients with unstable trochanteric fractures (AO/OTA 31 A2.1 - A2.3) were included in a retrospective parallel group comparative study conducted at R L Jalappa Hospital attached to Sri DevarajUrs Medical College, Tamaka, Kolar, Karnataka, India. The study protocol was approved by Institutional Ethics
Committee. (DMC/KLR/IEC - CER/33/2017 - 18)

A total of 54 patients, of which 27 each were operated with cemented bipolar hemiarthroplasty and PFN were selected for the study. All surgeries were performed within an average period of 4.2 days for BPA and 2.92 days for PFN. The mean age in BPA group was 67 years and 56 years PFN group.

Of the 27 fractures in BPA group, 18 were $31 \mathrm{~A} 2.3$ and 9 were 31A2.3, where-as in PFN group out of 27 cases, 9 were 31 A2.1, 14 were 31 A2.2 and 4 were 31 A2.3. The patients were operated in lateral decubitus position for BPA and supine on a fracture table for PFN. Antibiotic prophylaxis was followed (1gm Cefuroxime IV 30 minutes before procedure) as hospital protocols. All the cases were operated by the three lead surgeons of the hospital. The patients were allowed to bear weight as tolerated in cases of stable reduction in PFN group and $1^{\text {st }}$ post-operative day for BPA.

After clinical and radiological examination the clinical and functional outcomes were assessed in both groups using Harris hip score (HHS) at 4 weeks, 6 weeks, 12weeks, 6 months and 1 year on outpatient basis.

We classified the quality of reduction in PFN group as anatomical $(<5$ degrees of varus, valgus, anteversion or retroversion), acceptable (5-10 degrees) or poor $(>10$ degrees) ${ }^{[9]}$. A stem was considered to be unstable when there was progressive subsidence exceeding $3 \mathrm{~mm}$, any change in position, or a continuous radiolucent line wider than $2 \mathrm{~mm}$ at the bone-cement interface ${ }^{[1]}$. The fracture was considered to have healed when callus was visible and the patient did not feel any pain at the fracture site.

\section{Results}

The study included 27 patients in each group consisting 16 females and 11 males in BPA group whereas in PFN group there were 8 females and 19 males. In both groups mainly the mode of injury was self-fall. The patients in both the groups were comparable in their demographics as shown in table1.

The average blood loss was $310 \mathrm{ml}$ in BPA and $100 \mathrm{ml}$ in PFN which is statistically significant $(\mathrm{p}$-value $=0.001)$. The mean follow up of both the groups was 12 months. The time for surgery was shorter in PFN group (50 minutes) as compared to BPA (86 minutes) ( $\mathrm{p}$ value: 0.001). Mobilization was earlier in BPA group (3days). The average full weight bearing time in PFN group was 5 weeks. The average duration of surgery was shorter for PFN (50 minutes) as compared to BPA (86 minutes) $(\mathrm{p}$-value $=0.001)$.

The Functional outcome assessment of both these procedures were evaluated using Harris Hip Score is shown in table 2.The mean HHS, at 4 weeks of follow up was 77.81 for BPA and 71.18 for PFN (p-value $=0.001$ ), at 6 weeks of follow up was 79.88 for BPA and 72.81 for PFN (p-value $=0.001$ ). The mean HHS at 12 weeks of follow up was 81.77 for BPA and 74.74 for PFN (p-value $=0.001$ ), at 6 months of follow up was 83.63 for BPA and 75.25 for PFN (p-value $=0.001$ ) and at 1 year of follow up was 85.55 for BPA and 77.03 for PFN ( $\mathrm{p}$-value $=0.001)$. The radiographic pictures with follow up details are depicted in figure 1, 2 and 3.

We had complications related to surgery and prosthesis/implant in both groups. We had 4 superficial surgical site infections in BPA group and 2 patients had superficial surgical site infection in PFN group which were treated with appropriate antibiotics. In BPA group additionally 1 patient had deep infection, 2 patients had bed sores and 1 patient had DVT. All these complications were not seen in PFN group. One patient had Prosthetic related complications in BPA group where patient had dislocation of 
the prosthesis which was reduced closed.

Two patients had implant related complications in PFN group like distal locking screw loosening which was treated appropriately. One patient had abductor weakness in PFN group. One patient in the PFN group had died due reasons other than related to the surgery.

Table 1: Demographic, preoperative and postoperative data.

\begin{tabular}{|c|c|c|c|}
\hline Characteristics & Bipolar & PFN & P value \\
\hline Mean Age & 67 & 57 & 0.001 \\
\hline Sex (M:F) & $11: 16$ & $19: 8$ & 0.054 \\
\hline Mode (Fall:RTA) & $22: 5$ & $19: 3$ & 0.525 \\
\hline Side (R:L) & $17: 10$ & $14: 13$ & 0.528 \\
\hline Fracture Type (31a2.1:2.2:2.3) & $0: 9: 18$ & $8: 14: 5$ & - \\
\hline Mean TTS & 4.2 & 2.8 & 0.001 \\
\hline Mean BL & 309.259 & 101.852 & 0.001 \\
\hline Mean BTU & 1.333 & 0.111 & 0.001 \\
\hline Mean ST & 86 & 49.63 & 0.001 \\
\hline Mean LLD & 16 & 17.926 & 0.379 \\
\hline Mean BTOC & 0.87 & 0.11 & 0.001 \\
\hline Mean CTOW & 1.03 & 3.03 & 0.001 \\
\hline
\end{tabular}

M: Male; F: Female; RTA: Road Traffic Accident; R: Right; L: Left; TTS: Time To Surgery; BL: Blood Loss; BTU: Blood Transfusion Unit; ST: Surgery Time; LLD: Limb Length Discrepancy; BTOC: Bed To Chair; CTOW: Chair To Walking

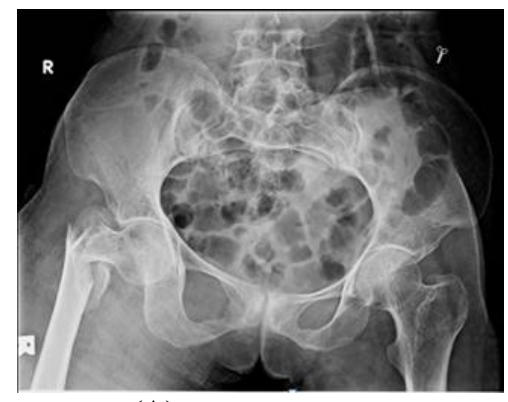

(A)

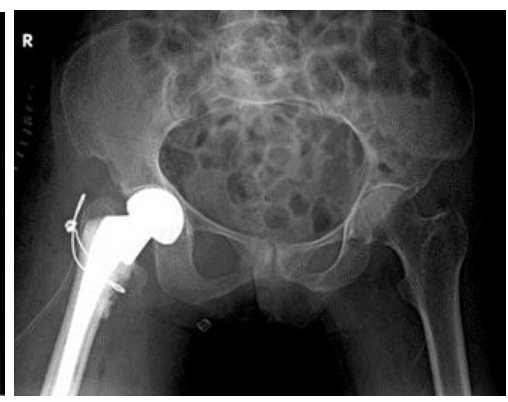

(B)

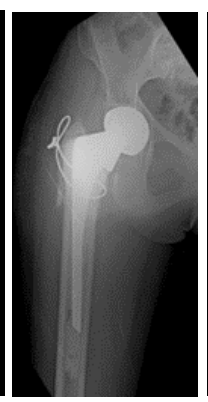

(C)

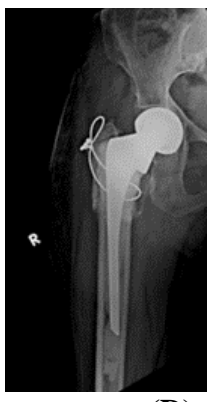

(D)

Fig 1: A: Pre-op radiographs IT fracture, B: Immediate post-op by cemented BA, C: 3 months post-op and D: 6 months post-op cemented BA.

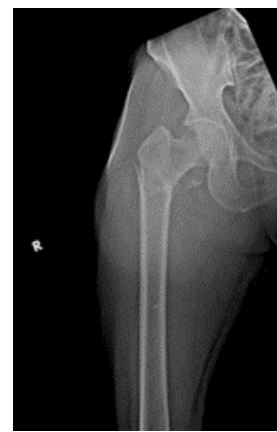

(A)

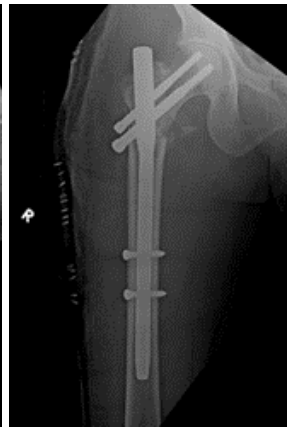

(B)

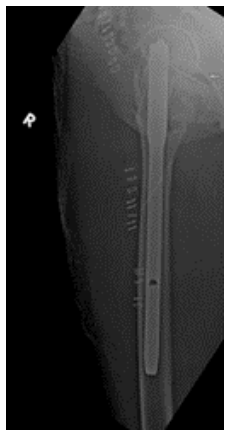

(C)

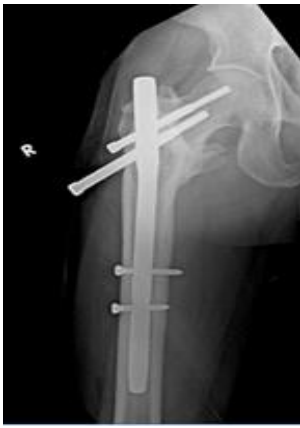

(A)

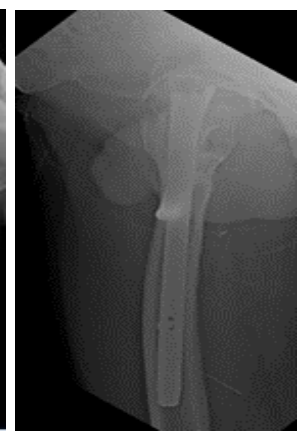

(B)

Fig 2: A: Pre-op radiographs IT fracture, B and C: Post-op anterioposterior and lateral views radiograph of $\mathrm{PFN}$

Fig 3: A and B: showing inferior lag screwback out (Z-effect) as a PFN complication.

Table 2: Functional outcome assessment using Harris Hip Score.

\begin{tabular}{|c|c|c|c|c|c|c|}
\hline & Group & No. & Mean & Std. Deviation & Std. Error Mean & P-value \\
\hline \multirow{2}{*}{ Post Op 4 Weeks } & BPA & 27 & 77.815 & 2.3540 & 0.4530 & \\
\cline { 2 - 7 } & PFN & 27 & 71.185 & 3.6903 & 0.7102 & 0.001 \\
\hline \multirow{2}{*}{ Post Op 6 Weeks } & BPA & 27 & 79.889 & 2.3912 & 0.4602 & \multirow{2}{*}{0.001} \\
\cline { 2 - 7 } & PFN & 27 & 72.815 & 3.9325 & 0.7568 & 0.4540 \\
\multirow{2}{*}{ Post Op 12 Weeks } & BPA & 27 & 81.778 & 2.3588 & 0.8070 & 0.001 \\
\cline { 2 - 7 } & PFN & 27 & 74.741 & 4.1933 & 0.6584 & \multirow{2}{*}{0.001} \\
\hline \multirow{2}{*}{ Post Op 6 Months } & BPA & 27 & 83.630 & 3.4211 & 0.8416 & \multirow{2}{*}{0.001} \\
\cline { 2 - 7 } & PFN & 27 & 75.259 & 4.3729 & 0.5266 & \\
\hline \multirow{2}{*}{ Post Op 1 Year } & BPA & 27 & 85.556 & 2.7363 & & \\
\cline { 2 - 7 } & PFN & 27 & 77.037 & 4.1276 & & \\
\hline
\end{tabular}

\section{Discussion}

Our study included 54 patients, 27 in each group and patients were followed up till 12 months post operatively. We found that the mean blood loss during BPA surgery was $310 \mathrm{ml}$ and during PFN surgery the blood loss was $100 \mathrm{ml}$ which is significantly lower $(\mathrm{p}$-value $=0.001)$. The number of blood 
units transfused during BPA (1.3 mean) were significantly more than PFN (0.1 mean) (p-value $=0.001)$. This is because of the invasive nature of the bipolar surgery resulting in higher blood loss in BPA compared to the less invasive surgery and closed reduction of fractures in PFN surgeries. The mean limb length discrepancy in BPA group was significantly higher compared to PFN group.

In elderly patients with osteoporotic fractures achieving and maintaining the reduction is a major problem during the fracture treatment. The implant related complications like cut through, screw back out are particularly more in this patient group. In our study we had two implant related complications which was comparable to the study done by Sameer et al. ${ }^{[12]}$. These complications are fewer in a cemented bipolar hemiarthroplasty. These implant related complications of PFN surgeries can be minimized by proper pre-operative planning and correct positioning of the screws.

The average duration of surgery in PFN group is shorter than BPA group considering the invasive nature of surgery of BPA group. Complications like surgical site infections including both superficial and deep infections were higher in the BPA group compared to the PFN group. These complications can also be attributed the duration of surgery and invasive nature of surgery. We had 5 superficial surgical site infection and 1 deep infection in BPA group which were treated appropriately as per the infection control guidelines which is comparable to the results of Görmeli et al ${ }^{[3]}$. The superficial and deep infections in the PFN group in our study were 2 and 0 respectively, these results are same as Sameer et al. ${ }^{[10]}$.

The average bed to chair mobilization time in BPA group was
1.03 days compared to 3.07 days in PFN group. The average chair to walker time in BPA group was 3 days and in PFN group was 5.18 weeks. These findings show that mobilization is much shorter in BPA group at each level which reduces overall hospital stay and complications related to it.In our study 2 patients of BPA group had bed sores and one patient had DVT which were treated conservatively, unlike the PFN group where these complications were not seen. These findings are similar to Görmeliet al. ${ }^{[3]}$ and Sameer et al. ${ }^{[10]}$ where these complications were either less or not seen in PFN group.

The BPA group consistently had statistically significant higher Harris Hip Scores compared to PFN group at all the follow ups up to one year. Harris hip scores were 85.55 and 77.03 in BPA and PFN groups, respectively. These findings are similar to study done by Rodop et al ${ }^{[12]}$, who reported the results of 37 patients with unstable intertrochanteric femoral fractures, who underwent cemented, bipolar hemiarthroplasty, were followed up for 12 months, and $80 \%$ of whom obtained excellent and good results using the Harris hip-scoring system. In a study by Tang et al. ${ }^{[13]}$. Harris hip scores were 83 and 80.2 in PFNA and hemiarthroplasty groups, respectively, with no statistically significant difference between the groups. In contrary to other studies, a study by Esen E et al. ${ }^{[14]}$ showed that the Harris hip score were 78.34 and 82.8 in BPA and PFN groups respectively.

The results of our study showed that both BPA and PFN are good treatment methods with better post operative outcomes in case of BPA which was also reported in a study by Sancheti et al. ${ }^{[15]}$.

Table 3: Comparison of complications with other studies.

\begin{tabular}{|c|c|c|c|c|}
\hline & Our study & Görmeli et al ${ }^{[3]}$ & Sameer et al. ${ }^{[10]}$ & Umuthataygölge et al. ${ }^{[11]}$ \\
\hline \multicolumn{5}{|l|}{ BPA } \\
\hline \multicolumn{5}{|l|}{ 1. Complications } \\
\hline A. Superficial surgical site infections & 5 & 4 & 0 & - \\
\hline B. Deep infection & 1 & 2 & 0 & 2 \\
\hline C. Pulmonary embolism & 0 & - & 2 & - \\
\hline D. Bed sores & 2 & 5 & 1 & - \\
\hline E. $\quad$ DVT & 1 & 6 & - & 3 \\
\hline F. Prosthetic related complications & 1 & - & 2 & 2 \\
\hline 2. Mortality at 12 months & 0 & 11 & 3 & - \\
\hline \multicolumn{5}{|l|}{ PFN } \\
\hline \multicolumn{5}{|l|}{ 1. Complications } \\
\hline A. Superficial surgical site infections & 2 & 5 & 2 & - \\
\hline B. $\quad$ Deep infection & 0 & - & 1 & 1 \\
\hline C. Pulmonary embolism & 0 & - & 0 & - \\
\hline D. Bed sores & 0 & 3 & 0 & - \\
\hline E. DVT & 0 & 4 & - & 0 \\
\hline F. Implant related complications & 2 & - & 2 & 5 \\
\hline 2. Mortality at 12 months & 1 & 6 & 2 & - \\
\hline
\end{tabular}

The limitation of this study is a shorter follow up period and that all the surgeries were not performed by same surgeon. Hence further long term follow up studies are required to compare the function outcomes of these two different procedures. The surgeon should choose an ideal method as per the individual case requirements.

\section{Conclusion}

Patients undergoing bipolar hemiarthroplasty had an earlier mobilization than patients treated with proximal femur nailing, but the surgical procedure time was shorter in the patients undergoing fixation with proximal femur nailing. Bipolar hemiarthroplasty is a better option in the treatment of unstable intertrochanteric fractures in osteoporotic elderly patients.

\section{References}

1. Dash SK, Sahoo PK, Panigrahi R, Kissan D, Das DS, Biswa MR. Cemented bipolar hemiarthroplasty versus proximal femoral nails: a prospective comparative outcome analysis in unstable elderly intertrochanteric fractures. Int J Health Sci Res. 2015; 5(6):99-106.

2. Emami M, Manafi A, Hashemi B, Nemati A, Safari S. Comparison of Intertrochanteric Fracture Fixation with Dynamic Hip Screw and Bipolar Hemiarthroplasty Techniques. Arch Bone Joint Surg. 2013; 1(1):14-17.

3. Görmeli G, Korkmaz MF, Görmeli CA, Adanaş C, Karataş T, Şimşek SA. Comparison of femur 
intertrochanteric fracture fixation with hemiarthroplasty and proximal femoral nail systems. Ulus Travma Acil Cerrahi Derg. 2015; 21(6):503-508.

4. Sonar UV, Ingle MV, Koichade MR, YelneAB, Malu N. Comparative study between trochanteric fixation nailing and cemented bipolar hemiarthroplasty for the treatment of unstable osteoporotic intertrochanteric neck femur fractures in elderly patients. IOSR J Dental Med Sci. 2014; 13(6):31-40.

5. Pal CP, Dinkar KS, Mittal V, Goyal A, Singh M, Hussain A. Role of bipolar hemiarthroplasty and total hip arthroplasty in unstable intertrochanteric fracture femur. J Orthop Allied Sci. 2016; 4:69-74.

6. Stappaerts KH, Deldycke J, Broos PL, Staes FF, Rommens PM, et al. Treatment of unstable peritrochanteric fractures in elderly patients with a compression hip screw or with a Vandeputte (VDP) endoprosthesis: a prospective randomised study. J Orthop Trauma. 1995; 9(4):292-297.

7. Gulati PS, Sharma R, Boparai RPS, Kapila R, Deewan A. Comparative study of treatment of intertrochanteric fractures of femur with long-stem bipolar prosthetic replacement versus dynamic hip screw fixation. $\mathrm{Pb}$ Journal of Orthopaedics. 2009; XI:1.

8. L Fan, X Dang, K Wang. Comparison between Bipolar Hemiarthroplasty and Total Hip Arthroplasty for Unstable Intertrochanteric Fractures in Elderly Osteoporotic Patients. PLoS One. 2012; 7(6):e39531.

9. Ozkan K, Unay K, Demircay C, Cakir M, Eceviz E. Distal unlocked proximal femoral intramedullary nailing for intertrochanteric femur fractures. International Orthopaedics. 2009; 33(5):1397-1400.

10. Mansukhani SA, Tuteja SV, Kasodekar VB, Mukhi SR. Management of Unstable Intertrochanteric Fractures using Three Different Modalities. Journal of Clinical and Diagnostic Research. 2017; 11(4):RC14-RC19.

11. Gölge UH, Pazarci O, Kilinç S, Nusran G, Kaymaz B, Göksel $\mathrm{F}$, et al. The treatment of intertrochanteric fractures comparison of PFN and hemiarthroplasty 3-year mortality study. Acta Orthopædica Belgica. 2016; 82(3):508-515.

12. Rodop O, Kiral A, Kaplan H, Akmaz I. Primary bipolar hemiprosthesis for unstable intertrochanteric fractures. IntOrthop. 2002; 26:233-7.

13. Tang P, Hu F, Shen J, Zhang L, Zhang L. Proximal femoral nail antirotation versus hemiarthroplasty: a study for the treatment of intertrochanteric fractures. Injury. 2012; 43:876-81.

14. Esen E, Dur H, Ataoğlu MB, Ayanoğlu T, Turanli S. Evaluation of proximal femoral nail-antirotation and cemented, bipolar hemiarthroplasty with calcar replacement in treatment of intertrochanteric femoral fractures in terms of mortality and morbidity ratios. Eklem Hastalik Cerrahisi. 2017; 28(1):35-40.

15. Sancheti KH, Sancheti PK, Shyam AK, Patil S, Dhariwal Q, Joshi R. Primary hemiarthroplasty for unstable osteoporotic intertrochanteric fractures in the elderly: A retrospective case series. Indian J Orthop. 2010; 44(4):428-34. 